\title{
Development of a Tool to Assess Barriers Faced by Children with Special Needs in Utilizing Dental Care Services
}

\author{
Krishnan Lakshmi ${ }^{1}$, Anusha $\mathrm{R}^{2}$, Kiran Iyer ${ }^{3}$, Madankumar P Diwakar ${ }^{4}$
}

\begin{abstract}
Aim: To develop and validate a questionnaire based on the Institute of Medicine (IOM) model of healthcare disparities to assess the barriers faced by children with special needs in utilizing dental care services, as perceived by their caregivers and dental professionals, in Chennai city.

Materials and methods: Based on the IOM model, two questionnaires were developed to assess barriers to dental care utilization from the dentist and caregiver's perspective. Content, face validation was done. Test-retest reliability was checked.

Results: Scale- Content validity index (S-CVI) value of 0.8 was obtained for both the scales. Cronbach's alpha value of 0.52 and 0.62 was obtained cfor the dentist and caregiver questionnaire, respectively.

Conclusion: The present study showed that the developed questionnaire was a reliable and valid tool to assess barriers faced by children with special needs in utilizing dental care services in an Indian context.

Clinical significance: The present study paves the way for further research on barriers encountered by children with special needs and its myriad implications in developing countries like India.
\end{abstract}

Keywords: Barriers, Caregivers, Cross-sectional, Dentist, Disabilities. Journal of Oral Health and Community Dentistry (2019): 10.5005/jp-journals-10062-0037

\section{INTRODUCTION}

T he Census of India in 2011 estimated that disability has affected nearly 1.2 million which approximates to about $2.21 \%$ of the total population of the country. Amongst these 29\% (46.2 lakhs) of them belong to children of the age group of less than 19 years. ${ }^{1}$ The term 'disabled' has undergone numerous changes in the epidemiological aspect rendering precise estimation of the affected population a tedious task. The pioneer inclusion of the disabled population in the census was made in 1872, following which it was dropped from 1991. In 2001 and 2011, 5 and 8 types of disabilities were included respectively. ${ }^{2}$ However, none of them were able to give a correct estimate of the actual burden has increased the disability-related burden. The implementation of 'Right to disability act' in 1995 with 7 disabilities which was later replaced in 2014 with 21 disabilities included under its domain. This modification in the term 'disability' over the years has, in turn, raised the count and global concern for children with special needs.

People with disability face a lot of health issues in their day to day life. This scenario may be explained by the relationship between individuals with disabilities and their environments. Penchansky and Thomas operationalized the concept in terms of "five As": affordability, availability, accessibility, accommodation and acceptability as the common factors influencing the utilization of healthcare services. ${ }^{3}$ Various human ecological models have examined the interactions by structuring the individual and the environment into four domains of influence that include (i) individual influences, which operate at the personal level and involve health, attitude and impairment, (ii) interpersonal influences, which involve social relationships with family, friends, and professionals, (iii) organizational level influence, which includes attributes of institutions within the community, such as programs and staff; and (iv) community influences, such as public transportation and the natural environment which involve the community-at-large. ${ }^{4}$ Although similar healthcare models have been designed using these $5 A^{\prime}$ 's as their basic framework, the IOM model of access to healthcare services, an extension of the Aday and Andersen model, specifically addresses the issue of equitable versus disparate
1,2Postgraduate Student, ${ }^{3}$ Reader, ${ }^{4}$ Professor and Head

${ }^{1-4}$ Department of Public Health Dentistry, Ragas Dental College, Chennai, Tamil Nadu, India

Corresponding Author: Krishnan Lakshmi, Postgraduate Student, Department of Public Health Dentistry, Ragas Dental College, Chennai, Tamil Nadu, India, e-mail: lakshmi.krish24@gmail.com

How to cite this article: Lakshmi K, Anusha R, lyer K, Diwakar MP. Development of a Tool to Assess Barriers Faced by Children with Special Needs in Utilizing Dental Care Services. J Oral Health Comm Dent 2019;13(1):1-4.

Source of support: Nil

Conflict of interest: None

healthcare. This model especially addresses the structural, financial and personal barriers in the equitable use of healthcare services and the resulting health outcome. Literature reveals that people with disabilities are more likely to have unmet healthcare needs and use fewer medical services associated with preventive care or health maintenance. ${ }^{3}$ Keeping these in consideration, for these children oral health is far from their priority, as it is always dependent on their general health and oral hygiene maintenance by their caregiver. Though plenty of cross-sectional studies have reported barriers faced in dental care delivery among disabled children, there are hardly any available standardized questionnaires for assessing barriers faced by disabled children in utilizing dental care. ${ }^{5-8}$ Thus the aim of this study was to develop and validate a questionnaire based on the IOM model of healthcare disparities to assess the barriers faced by children with special needs in utilizing dental care services, as perceived by their caregivers and dental professionals, in Chennai city.

\section{Materials and methods}

\section{Questionnaire Development}

Based on the IOM model, two questionnaires were developed. One questionnaire consisted of 15 items including, the demographic

(c) The Author(s). 20190pen Access This article is distributed under the terms of the Creative Commons Attribution 4.0 International License (http://creativecommons. org/licenses/by/4.0/), which permits unrestricted use, distribution, and non-commercial reproduction in any medium, provided you give appropriate credit to the original author(s) and the source, provide a link to the Creative Commons license, and indicate if changes were made. The Creative Commons Public Domain Dedication waiver (http://creativecommons.org/publicdomain/zero/1.0/) applies to the data made available in this article, unless otherwise stated. 
details, the pattern of utilizing dental care services, architectural limitations of dental clinics, cultural and financial barriers faced in the utilization of dental care services. This was administered to the caregivers of children with special needs. Another 17 items questionnaire to assess the dental practitioners' perspective was developed, which included demographic details, barriers encountered in treating children with special needs and attitude based questions. A total of 32 closed-ended questions were assessed which were predominantly of Yes/No type or had multiple options form which the respondents had to choose from. The former questionnaire addressing the caregivers' perspective was also translated to the regional language (Tamil) and then backtranslated to English with the help from Tamil and English scholar, to ensure consistency.

\section{Study Design}

A cross-sectional study was conducted to assess the validity and reliability of these questionnaires. Participants in the study included, parents/caregivers of children who spend more than 15 hours/ day with the disabled children and private dental practitioners of Chennai city who had previous experiences of treating at least one disabled child in his/ her clinic in the recent times (within past 3 months). Private dental care practioners who were members of a Tamil Nadu Dental Council were only selected for this study. There was no restriction on the type of disability which the children of the participants had or the participating doctors faced in their practice. It ranged from visual impairment, hearing impairment, cerebral palsy with mental retardation, physically handicapped, autism, ADHD to any form of intellectual disability as clinically diagnosed and under treatment at a tertiary care center. The study sample consisted of 100 female caregiver of disabled children aged between 3 years and 15 years and 195 private dental practitioners in Chennai city. The protocol was approved by the Institutional Review Board. Verbal informed consent was obtained from the participants. Privacy and confidentiality of the information obtained was maintained throughout the study.

\section{Validating Procedure}

The questionnaires were assessed for the following validity:

\section{Content Validity}

Content validity was undertaken to ascertain whether the content of the questionnaire was appropriate and relevant to the study purpose. Content validity indicates whether the content reflects a complete range of the attributes under study and is usually undertaken three or more experts. ${ }^{9}$ To estimate the content validity of the questionnaire, the principal investigator clearly defines the objectives of the study and explain the conceptual framework of the questionnaire using literature reviews. Once the questionnaire is established, and the conceptual framework becomes clear six experts from the field namely 4 pedodontist who deal with special needs children, one dental practitioner and one general practitioner who deal with special children on day to day basis were chosen purposefully to review the drafted questionnaires and ensure its consistency with the concept developed. Each reviewer independently rated the relevance of each item on the questionnaire to the conceptual framework using a 3 point Likert scale ( 1 = essential, 2 = essential, but not useful, 3 = not useful). The content validity index (CVI) was used to estimate the validity of the individual items and scale. ${ }^{10}$

\section{Face Validity}

Face validity indicates the questionnaire appears to be appropriate to the study purpose and content area. It is the easiest validation process to undertake. It evaluates the appearance of the questionnaire in terms of feasibility, readability, consistency of style and formatting, and the clarity of the language used. ${ }^{11-13}$ Thus, face validity is a form of usability rather than reliability. To determine the face validity of our questionnaires, an evaluation form was developed to help respondents assess each question in terms of:

- The clarity of the wording,

- The likelihood the target audience would be able to answer the questions,

- The layout and style.

Twenty general dental practitioners from private clinics of Chennai city and caregivers of children with special needs attending a tertiary care center of Chennai city were randomly selected and completed the face validity form on a Likert scale of $1-4$, strongly disagree $=1$, disagree $=2$, agree $=3$, and strongly agree $=4$.

\section{Construct Validity}

Construct validity refers to the degree to which the items on an instrument relate to the relevant theoretical construct. ${ }^{13,14}$ Construct validity is a quantitative value rather than a qualitative distinction between 'valid' and 'invalid'. It helps to assess the extent to which a measuring instrument accurately measures a theoretical construct it is designed to measure. Measured by correlating performance on the test with performance on a test for which construct validity has already been done. To measure construct validity usually factor analysis is done. Since our questionnaire was predominantly knowledge, attitude-based to assess the barriers faced and the outcomes were only categorical, which required the subjects to choose 1 statement best reflecting their opinions from a list of mutually exclusive options with no inherent order, construct validity was not assessed. This type of question does not lend itself readily to quantification, and hence factor analysis was not performed by the investigators.

It was also ensured by the investigators that the sample of participants who were included to assess the face validity was entirely different from the pilot population for whom these questionnaires were administered to assess the reliability.

\section{Reliability}

Once the validity procedures were completed, the final draft of the questionnaire was examined to assess its reliability. Reliability refers to the ability of a questionnaire to consistently measure an attribute and how well the items fit together, conceptually. ${ }^{4,6}$ Although reliability is necessary, is not sufficient to validate an instrument, because an instrument may be reliable but not valid. ${ }^{13,15,16}$ Cronbach and Shavelson ${ }^{17}$ suggested researchers should consider the following issues when determining reliability:

- Stability of the questionnaire

- Internal consistency

- Equivalence.

Two estimators of reliability commonly used, internal consistency reliability and test-retest reliability were both used to assess the reliability of the questionnaire. The questionnaires were administered to the randomly selected sample of 30 dentists and 30 parents/caregivers twice within an interval of 4 weeks to determine the reliability. 


\section{ResUlts}

The English version of the drafted questionnaire for caregivers was translated to Tamil back-translated to English and was found to match with the original English version.

\section{Content Validity}

According to the $\mathrm{CVI}$ index, a rating of three or four indicated invalid content and this was found to be consistent with the conceptual framework. $^{10}$

Among the 32 questions assessed ( 15 for parents/caregivers and 17 for dentists), 2 of them (questions 1 and 2) for dental practitioners were deemed to be invalid because they yielded CVIs of $4 / 6=0.6$ and were henceforth removed from the final draft.

All the remaining items were valid with CVIs ranging from $0.7(5 / 6)$ to $0.100(6 / 6)$ and were retained. S-CVI for both the questionnaire was 0.8 which is said to be highly acceptable (Table 1).

\section{Face Validity}

A rating of three or four on a 4-point Likert scale for each question by the respondents is considered acceptable. Ninety-five percent of the responded dentists indicated, they understood the questions and found them easy to answer, and 90\% indicated the appearance and layout would be acceptable to the intended target audience.

In case of the questionnaire for caregivers $95 \%$ indicated that they did not understand question 4 which stated "Which among the following is reflected by your child due to oral health problem according to OHIP scale", therefore this question was reframed as "Which among the following is reflected by your child due to oral health problem"

\section{Test-retest}

Test re-test reliability was tested among a pilot sample of 30 dental practitioners and 30 caregivers of children with special needs in a period of 4 weeks. The correlation ranged from $78-85 \%$.

\section{Discussion}

The purpose of this study was to determine the validity and reliability of the questionnaire developed to assess the barriers encountered by children with special needs in utilizing dental care services.

The validity of the questionnaire was determined by calculating the Content Validity Index of each item and scale which was found to be in the acceptable range (0.7-1.00) and the two questions which had a very low value of 0.4 , and hence, removed from the final draft.

The modified English version of the questionnaire was translated to the regional language (Tamil) and back-translated to English. It was found to be consistent with the original version. The test re-test reliability of a questionnaire is greatly dependent on the interim period between the days of the administration of the questionnaire. If it is too short, memory skills will influence the response and yield confounding results. In case of a longer time interval, the attribute being examined might get changed, and low correlation may indicate this change rather than poor reliability. ${ }^{18}$ In our present study, a period of 4 weeks was ensured between the 2 consecutive administrations, as it was neither too short nor too long.

Our research assessed a variety of barriers faced by dental practitioners and caregivers of special children according to the IOM model for healthcare utilization. These were consistent with the findings of Thompson et al. who stated that affordability, availability, accommodation, accessibility, and acceptability are

Table 1: Depicting the content validation of each item of both questionnaire

\begin{tabular}{|c|c|c|c|c|c|c|c|c|c|c|c|c|}
\hline \multirow[b]{2}{*}{ Items } & \multicolumn{2}{|c|}{ Expert 1} & \multicolumn{2}{|c|}{ Expert 2} & \multicolumn{2}{|c|}{ Expert 3} & \multicolumn{2}{|c|}{ Expert 4} & \multicolumn{2}{|c|}{ Expert 5} & \multicolumn{2}{|c|}{ Expert 6} \\
\hline & $P Q$ & $D Q$ & $P Q$ & $D Q$ & $P Q$ & $D Q$ & $P Q$ & $D Q$ & $P Q$ & $D Q$ & $P Q$ & $D Q$ \\
\hline $\mathrm{Q} 1^{*}$ & 1 & 1 & 1 & 1 & 1 & 2 & 1 & 2 & 1 & 2 & 1 & 2 \\
\hline Q2* & 1 & 2 & 1 & 2 & 1 & 2 & 1 & 2 & 1 & 1 & 2 & 2 \\
\hline Q3 & 1 & 1 & 1 & 1 & 1 & 1 & 1 & 1 & 1 & 1 & 1 & 1 \\
\hline Q4 & 1 & 2 & 1 & 1 & 1 & 1 & 2 & 1 & 1 & 1 & 1 & 1 \\
\hline Q5 & 1 & 1 & 1 & 1 & 1 & 1 & 1 & 1 & 1 & 1 & 1 & 1 \\
\hline Q6 & 1 & 2 & 1 & 1 & 1 & 1 & 1 & 1 & 1 & 1 & 1 & 1 \\
\hline Q7 & 1 & 2 & 1 & 1 & 1 & 1 & 1 & 1 & 1 & 1 & 1 & 1 \\
\hline Q8 & 1 & 1 & 1 & 1 & 1 & 1 & 1 & 1 & 1 & 1 & 1 & 1 \\
\hline Q9 & 1 & 1 & 1 & 1 & 1 & 1 & 1 & 1 & 1 & 1 & 1 & 1 \\
\hline Q10 & 1 & 1 & 1 & 1 & 1 & 1 & 1 & 1 & 1 & 1 & 1 & 1 \\
\hline Q11 & 1 & 1 & 1 & 1 & 1 & 1 & 1 & 1 & 1 & 1 & 1 & 1 \\
\hline Q12 & 1 & 1 & 1 & 1 & 1 & 1 & 1 & 1 & 1 & 1 & 1 & 1 \\
\hline Q13 & 2 & 1 & 1 & 1 & 1 & 1 & 1 & 1 & 1 & 1 & 1 & 1 \\
\hline Q14 & 1 & 1 & 1 & 1 & 1 & 1 & 1 & 1 & 1 & 1 & 1 & 1 \\
\hline Q15 & 1 & 1 & 1 & 1 & 1 & 1 & 2 & 1 & 1 & 1 & 1 & 1 \\
\hline Q16 & - & 1 & - & 1 & - & 1 & - & 1 & - & 1 & - & 1 \\
\hline Q17 & - & 1 & - & 1 & - & 1 & - & 1 & - & 2 & - & 2 \\
\hline
\end{tabular}

DQ-Dentist questionnaire PQ-Parent/caregiver questionnaire

$1=$ Highly relevant, relevant

$2=$ Somewhat relevant, not relevant

* = Questions removed due to low CVI. 
the major barriers faced by patients with disabilities in access to health care. ${ }^{3}$

Similar studies by to assess parental perceptions on the barriers faced by children with special needs by Gerreth, ${ }^{6}$ Linda et al., Sharifa ${ }^{8}$ and Bhaskar et al. ${ }^{19}$ have reported that availability of dental services, dental fear, cost, income, accessibility to dental clinic are few major barriers encountered in accessing dental care services. The dentist perception of barriers faced by disabled children in accessing dental services was reported by Adyanthaya et al. ${ }^{20}$ as the lack of trained practitioners and motivation of caregivers as the major determinants. Although there are pieces of evidence regarding the barriers faced there is a lack of a validated tool based on a healthcare model to assess the same among both practitioners and caregivers, which is present in our study. The IOM model was selected because it specifically addresses the equitable and disparate healthcare with respect to both the health status of the individual and available healthcare services.

In our present study, participants were comfortable filling in their responses and there was no ambiguity of words or phrases. Although question 4 which mentioned about oral health-related quality of life, the participants found it hard to understand. Hence it was modified to simpler terms to facilitate easy understanding. Question 3 regarding the 'Right to Disability Act 2016' was mostly left unanswered indicating the lack of awareness among the dental practitioners. This implies the need to update the knowledge among doctors to provide the right care to the patients in need at the right time.

The overall reliability of the questionnaire administered to the dentist and caregivers was 0.56 and 0.62 , respectively. This low value could be because of the questions in the questionnaire were predominantly knowledge-based and also the educational qualification among caregivers was not uniform leading to varying levels of awareness.

The limitation of this study is that the findings cannot be generalized to the adult population and the scale developed was more subjective. Also, all the questions were closed-ended expecting the respondents to choose from the given options limiting the expression of participants' perspectives. As the questions were predominantly knowledge-based factor analysis could not be performed. Further studies are recommended to analyze the specific barriers with respect to each type of disability and further address them to provide achieve health for all.

Though several studies have used a questionnaire to understand the barriers faced, there is no standardized tool present to assess the barriers encountered by special children. To the best of our knowledge, this was the first study that has attempted to check the validity and reliability of the self-developed questionnaire on barriers faced by children with special needs using a healthcare utilization model among the caregivers and dental practitioners of south India.

\section{Conclusion}

The present study showed that the developed questionnaire is a reliable and valid tool in an Indian context. Results indicate that the developed questionnaires have good psychometric properties when tested among the dentist and caregivers populations in India.

\section{Clinical significance}

The present study paves the way for further research on barriers encountered by children with special needs and its myriad implications in developing countries like India.

\section{References}

1. Ministry of statistics and program implementation. Disabled population in India: A statistical profile 2016 March: Retrieved from: http://:mospi.nic.in/sites/default/files/publication.../Disabled_ persons_in_India_2016.pd.

2. Dimension of disability in India. (2016) Disabled Persons in India: A statistical profile 2016. [Online]. Ministry of Statistics and Programme. Available from http://mospi.nic.in/sites/default/files/publication reports/Disabled_persons_in_India_2016.pdf [Last accessed May 2019].

3. Michelle AM, Elham M, Shoou-Yih L. The intersection of disability and healthcare disparities: a conceptual framework. Disabil Rehabil 2015;37(7):632-641.

4. Vijay V, James HR, Frederick K. Development of the Barriers to Physical Activity Questionnaire for People with Mobility Impairments. Disabil Rehabil 2015;8(4):547-556.

5. Yasir IM, Shoaib R, Hamaza HM. Factors that limit access to dental care for person with disabilities. Pak Armed Forces Med J 2016; 66(2): 230-234.

6. Gerreth K, Lewcika BM. Acess Barriers to Dental Health Care in Children with Disability, A Questionarie Study of parents. JARID 2015; 29:139-145.

7. Linda NP, Getzin A, Graham D, et al. Unmet Dental Needs and Barriers to Care for Children with Significant Special Health Care Needs, Pediatric dentistry 2011;13(1):29-36.

8. Sharifa AM. Access to dental care for persons with disabilities in Saudi Arabia (Caregivers' perspective) Journal of Disability and Oral Health 2012;13(2):51-61.

9. Lawshe CH. A Quantitiative Approach to Content Validity.Personnel Psycology 1975;28:563-575.

10. Lynn MR. Determination and quantification of content validity. Nursing Research 1996;35:382-385.

11. Haladyna T. Developing and Validating multiple-choice test items.1999 New Jersey: Lawrence Erlbaum.

12. Trochim WMK. The Research Methods Knowledge Base 2001 Cincinnati: Atomic Dog.

13. DeVon HA, Block ME, Moyle-Wright P, et al. A psychometric Toolbox for testing Validity and Reliability. Journal of Nursing scholarship, 2007;39(2):155-164.

14. Kane M. Current concerns in validity theory. Journal of Educational Measurement 2001;38: 319-342.

15. Beanland C, Schneider Z, LoBiondo-Wood G, et al. Nursing research: Methods, critical appraisal and utilization. 1999.

16. Pilot D, Hunger B. Nursing research: principals and method 1999; Philadelphia: Lippincott Williams and Wilkins.

17. Cronbach LJ, Shavelson RJ. My Current Thoughts on Coefficient Alpha and Successor Procedures Educational and Psychological Measurement 2004;64(3):391-418.

18. Pavithra R, Lalithambigai G, Junaid M, et al. Content Validity and Reliability of the 13-Item Sense of Coherence Scale among 13-15 Year Old School Children in Chennai City. The International Journal of Indian Psycology 2015;3(1):43-50.

19. Bhaskar VB, Janakiram C, Joseph J. Access to Dental Care among Differently Disabled Children in Kochi, Journal of Indian Association of Public Health dentistry 2016;14(1):29-34.

20. Adhyanthaya A, Sreelakshmi N, Ismail S, et al. Barriers to dental care for children with special needs: General dentists' perception in Kerala, India. J Indian Soc Pedod Prev Dent 2017;35:216-222. 